\title{
Original
}

\section{A Comparative Study on the Morphological Changes in the Pharyngeal Airway Space before and after Sagittal Split Ramus Osteotomy}

\author{
Tomoko Uozumi, ${ }^{1,2)}$, Yoshiyasu Yoshikawa ${ }^{2)}$, Yukiko Yokoi' ${ }^{3)}$ Nobuyuki Ando ${ }^{3)}$, Akira Taguchi'), \\ Tadashi Ogasawara ${ }^{4)}$, Nobuyuki Udagawa ${ }^{1)}$ and Norimasa Okafuji ${ }^{1,3)}$ \\ ${ }^{1)}$ Department of Hard Tissue Research, Graduate School of Oral Medicine, Matsumoto Dental University, Shiojiri, Japan \\ 2) Yoshikawa Orthodontic Clinic, Osaka, Japan \\ ${ }^{3)}$ Center for Excellence in Teaching and Learning, Matsumoto Dental University, Shiojiri, Japan \\ 4) Department of Oral Health Promotion, Graduate School of Oral Medicine, Matsumoto Dental University, Shiojiri, Japan \\ (Accepted for publication, October 1, 2011)
}

\begin{abstract}
This study investigated the difference in pharyngeal spaces in orthodontic patients with or without setback surgery. The control group consisted of 28 patients who underwent orthodontic treatment alone and a study group consisting of 53 patients that had orthodontic treatment and sagittal split ramus osteotomy. The pharyngeal spaces were compared in both groups using cephalogram before and after treatment. During the initial visit, the middle and lower pharyngeal spaces were significantly larger in the study group than in the control group. However, there were no significant differences in any parameter used to measure the pharyngeal space between the two groups after surgery. The surgery contributed to the reduction in the size of the pharyngeal spaces. The pharyngeal space of patients who need setback surgery may be larger than orthodontic patients who do not need surgery.
\end{abstract}

Key words: Maxillofacial deformity, Pharyngeal space, Mandibular prognathic surgery, Malocclusion, Orthodontic therapy

\section{Introduction}

Changes in the pharyngeal airway in patients with skeletal mandibular prognathism after sagittal split ramus osteotomy (SSRO) have been considered due to the narrowing of the pharyngeal airway postoperatively ${ }^{1-11)}$. Emata et al. ${ }^{1)}$ examined the effects of surgical orthodontic treatment in patients with skeletal mandibular protrusion and reported the antero-posterior decreased in the diameter of the airway in patients with mild setback of the mandible.

Moreover, Yoshida et al. ${ }^{8)}$ reported that patients treated with SSRO were recognized to have mild narrowing of the upper part of the pharyngeal airway but significant narrowing of the lower pharyngeal airway after treatment with skeletal mandibular protrusion was not observed. Eggendperger et al reported that after the initial decrease in pharyngeal airway post-operatively, the oropharyngeal airway continued to decrease even after more than 12 years post-operatively ${ }^{10)}$.

Recent reports mentioned about the side effects in breathing

Correspondence to: Prof. Norimasa Okafuji, DDS, PhD, Department of Hard Tissue Research, Graduate School of Oral Medicine, Matsumoto Dental University, 1780 Hirooka-Gobara, Shiojiri, Nagano, 399-0781 Japan, Tel and Fax: +81-263-51-2335, E-mail: okafuji@po.mdu.ac.jp during sleep brought about by the narrowing of pharyngeal airway after mandibular setback surgery ${ }^{12-14)}$.

On the other hand, some reports pointed out that patients with skeletal mandibular protrusion have larger airway than normal pharyngeal airway ${ }^{15}$. From our years of clinical experience, it has been considered that patients with skeletal mandibular protrusion have wider pharyngeal airway than those with normal occlusion. However, no studies have been done comparing the pharyngeal airway in patients with skeletal mandibular protrusion and those with normal occlusion.

In this study, we attempted to validate the hypothesis whether patients with skeletal mandibular protrusion have narrower pharyngeal airway after mandibular setback. For this, the size of the pharyngeal airway in patients with skeletal mandibular protrusion (1) before surgery and (2) post-operatively were compared with patients treated with general orthodontics only as control.

\section{Materials and methods}

Of the 439 patients who were diagnosed with jaw deformity at a certain orthodontic clinic from June 30, 1998 to June 30, 2010, 257 cases were selected. Those patients regardless of gender were 
treated with SSRO and the pre and post-operative data were obtained.

The research was designed so as to have an equal number of male and female orthodontic patients. However, the study was only conducted among female patients since there were more female than male patients. For some female patients, the retrusion intended for SSRO exceeded $10 \mathrm{~mm}$ and so those patients were excluded in the study. The target asymmetry of jaw deformation was less than $5 \mathrm{~mm}$. A total of 53 adult female patients (26 Y1M \pm $6 \mathrm{Y} 1 \mathrm{M}$ ) with an asymmetry of less than $5 \mathrm{~mm}$ were eventually chosen for the study group.

For the control group, 28 adult female patients (28 Y $1 \mathrm{M} \pm 12$ Y 4M) out of 196 patients without skeletal disharmony treated with orthodontics alone were used in the study. In addition, not all patients were adults or of legal age. Minors were also included in the study due to the growth and development which have been completed.

Standard $\mathrm{x}$-ray photographs of the control and the study groups were immediately taken (not exceeding cephalogram) above the hypopharynx and these data were evaluated. For the study group, 3 lateral cephalograms were taken which were during the initial visit, at the end of active orthodontic treatment and after SSRO. For the control group, 2 lateral cephalograms were taken namely during the initial visit and at the end of active orthodontic treatment.

The photographs of the lateral cephalogram were taken while the patient was standing, positioned in the Frankfurt plane parallel to the floor. While photographs were taken, the patients were asked to breathe through the nose with the tongue positioned at rest and was instructed to bite in centric occlusion.

Evaluation of the size of the pharyngeal airway was performed in accordance with Mochida et al. ${ }^{16)}$

\section{Lateral cephalogram measurement}

The following items were measured from cephalometric tracings on each side in the control and study groups (Figure 1).

1) Measurement of the size of the pharyngeal airway ( $\mathrm{mm})$

(1) Palatal pharyngeal space (PPS): the anteroposterior depth of the pharynx measured between the posterior pharyngeal wall and the PNS on a line parallel to the FH plane that runs through the PNS.

(2) Superior Posterior pharyngeal space (SPPS): the anteroposterior depth of the pharynx measured between the posterior pharyngeal wall and the dorsum of the soft palate on a line parallel to the FH plane that runs through the middle of the line from the PNS to tip of the soft plate.

(3) Middle pharyngeal space (MPS): the anteropostesterior depth of the pharynx measured between the posterior pharyngeal wall and the dorsum of the tongue on a line parallel to the $\mathrm{FH}$ plane that runs through tip of the soft plate.

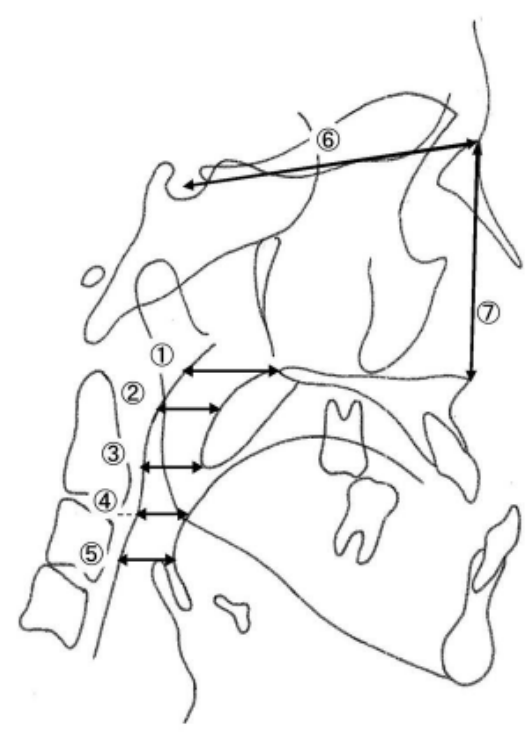

Figure 1. Lateral cephalogram

(1) PPS (2) SPPS (3) MPS (4) IPS (5) EPS (6) S-N (7) N-ANS

(4) Inferior pharyngeal space (IPS): the anteroposterior depth of the pharynx measured between the posterior pharyngeal wall and the surface of the tongue on a line parallel to the FH plane that runs through the most anterior point on the second cervical vertebra.

(5) Epiglottic pharyngeal space (EPS): the anteroposterior depth of the pharynx measured between the posterior pharyngeal wall and the surface of the tongue on a line parallel to the $\mathrm{FH}$ plane that runs through the tip of the epiglottis.

2) Measurements of skeletal indicators (mm)

(6) S-N: Sella-nasion distance

(7) N-ANS: Nasion-anterior nasal spine distance

\section{Data analysis}

Data analysis for each was performed as follows:

1) Difference in the size of the pharyngeal airway in control and study groups at the initial visit

Skeletal indicators were examined by analysis of covariance (ANCOVA) corrected for age (S-N, N-ANS) at the initial visit.

2) Difference in the size of the pharyngeal airway in control and study groups at the end of active treatment

Skeletal indicators were examined by ANOVA corrected for age at the initial visit.

3) Difference in the size of the pharyngeal airway before and after treatment in the control group

Comparison was carried out using paired T-test.

4) Difference in the size of the pharyngeal airway before and after treatment in the study group

At the initial visit, comparison of the pre- and post-operative active treatment was examined by one-way repeated ANOVA. This analysis was adjusted for age and duration of treatment as a 
Tomoko Uozumi et al.: Morphological Changes in the Pharyngeal Airway Space

Table 1. Comparison between the control and study groups during the initial visit

\begin{tabular}{lllr}
\hline & $\begin{array}{l}\text { Control } \\
\text { group }(\mathrm{n}=28)\end{array}$ & $\begin{array}{l}\text { Study } \\
\text { group }(\mathrm{n}=53)\end{array}$ & \\
\hline PPS & $28.0 \pm 0.5$ & $28.7 \pm 0.4$ & $\mathrm{p}=0.149$ \\
SPPS & $12.7 \pm 0.7$ & $14.5 \pm 0.5$ & $\mathrm{p}=0.047$ \\
MPS & $11.1 \pm 0.6$ & $12.4 \pm 0.4$ & $\mathrm{p}=0.064$ \\
IPS & $12.1 \pm 0.6$ & $14.3 \pm 0.5$ & $\mathrm{p}=0.005$ \\
EPS & $11.7 \pm 0.6$ & $13.6 \pm 0.4$ & $\mathrm{p}=0.016$ \\
\hline & \multicolumn{4}{c}{ Mean $\pm \mathrm{SE}$} & (mean \pm SE) & $\mathrm{SE}:$ standard error
\end{tabular}

Table 2. Comparison between the control and study groups after treatment

\begin{tabular}{llll}
\hline & $\begin{array}{l}\text { Control } \\
\text { group }(\mathrm{n}=28)\end{array}$ & $\begin{array}{l}\text { Study } \\
\text { group }(\mathrm{n}=53)\end{array}$ \\
\hline PPS & $28.4 \pm 0.5$ & $29.0 \pm 0.4$ & $\mathrm{p}=0.379$ \\
SPPS & $12.8 \pm 0.6$ & $12.5 \pm 0.4$ & $\mathrm{p}=0.731$ \\
MPS & $11.0 \pm 0.5$ & $10.6 \pm 0.4$ & $\mathrm{p}=0.490$ \\
IPS & $11.8 \pm 0.6$ & $11.5 \pm 0.5$ & $\mathrm{p}=0.723$ \\
EPS & $11.6 \pm 0.6$ & $11.7 \pm 0.4$ & $\mathrm{p}=0.934$ \\
\hline & & & Mean \pm SE
\end{tabular}

Table 3. Comparison in the control group

\begin{tabular}{lcccl}
\hline & T0:Pre-treatment & T1:Post-treatment & T-test \\
\hline PPS & $28.0 \pm 0.5$ & $28.6 \pm 0.5$ & $\mathrm{p}=0.032$ & $*$ \\
SPPS & $12.8 \pm 0.6$ & $12.8 \pm 0.6$ & $\mathrm{p}=0.972$ & NS \\
MPS & $11.2 \pm 0.5$ & $11.2 \pm 0.5$ & $\mathrm{p}=0.978$ & $\mathrm{NS}$ \\
IPS & $12.2 \pm 0.7$ & $12.0 \pm 0.7$ & $\mathrm{p}=0.518$ & NS \\
EPS & $11.9 \pm 0.6$ & $11.7 \pm 0.7$ & $\mathrm{p}=0.660$ & NS \\
\hline
\end{tabular}

$\mathrm{n}=28$; mean $\pm \mathrm{SE}$; NS: not significant; ${ }^{*} \mathrm{P} \prec 0.05$

covariate during the initial visit. Multiple comparisons were adjusted by means of Bonferroni.

All statistical analyses were considered to be significant at 5\% level. All computations were done using SPSS version 8 (Chicago, Il). The study was approved by the Ethics Committee of Matsumoto Dental University on October 31, 2010.

\section{Results}

Difference in the size of the pharyngeal airway between the control group and study group during the initial visit

After measuring the size of the pharyngeal airway (mm) of the control and the study group at the initial visit, SPPS, IPS and EPS were significantly higher in the study group compared to the control group. An increased tendency in MPS was also observed in the study group. No significant difference in PPS which corresponds to the nasopharynx was observed (Figure 2, Table 1).

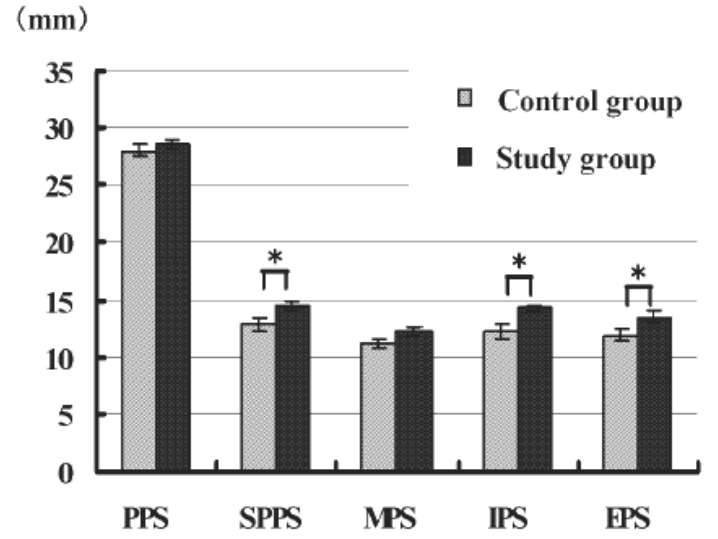

Figure 2. Control group versus the study group during the initial visit (ANCOVA -Analysis of covariance) $* \mathrm{P}<0.05$

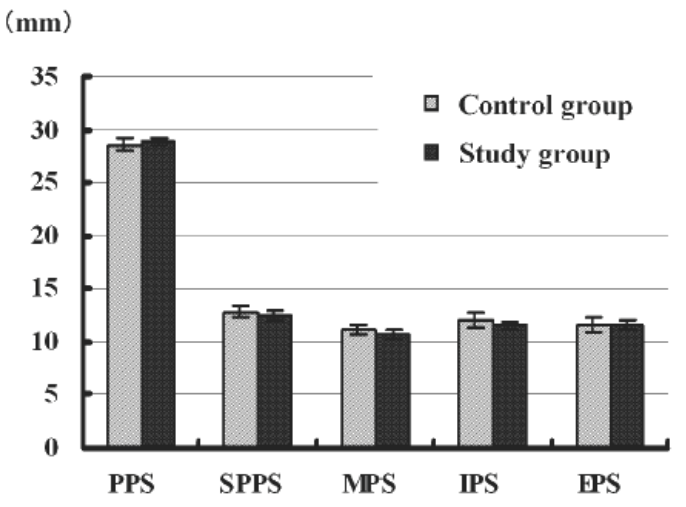

Figure 3. Control group versus the study group after treatment Age was adjusted by analysis of covariance with skeletal indicators (ANCOVA -Analysis of covariance) $* \mathrm{P}<0.05$

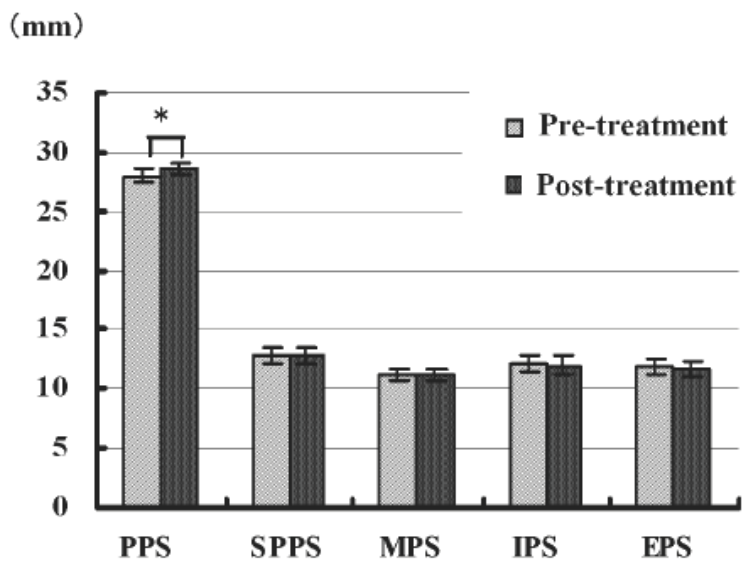

Figure 4. During the initial visit after treatment compared to the control group Paired T-test, $\quad * \mathrm{P}<0.05$

\section{Difference in the size of the pharyngeal airway between the} control and the study group at the end of the treatment

There was no significant difference in PPS, SPPS, MPS, IPS and EPS between the control and study group regarding the size of the pharyngeal airway (mm) at the end of active treatment 


\section{PPS}
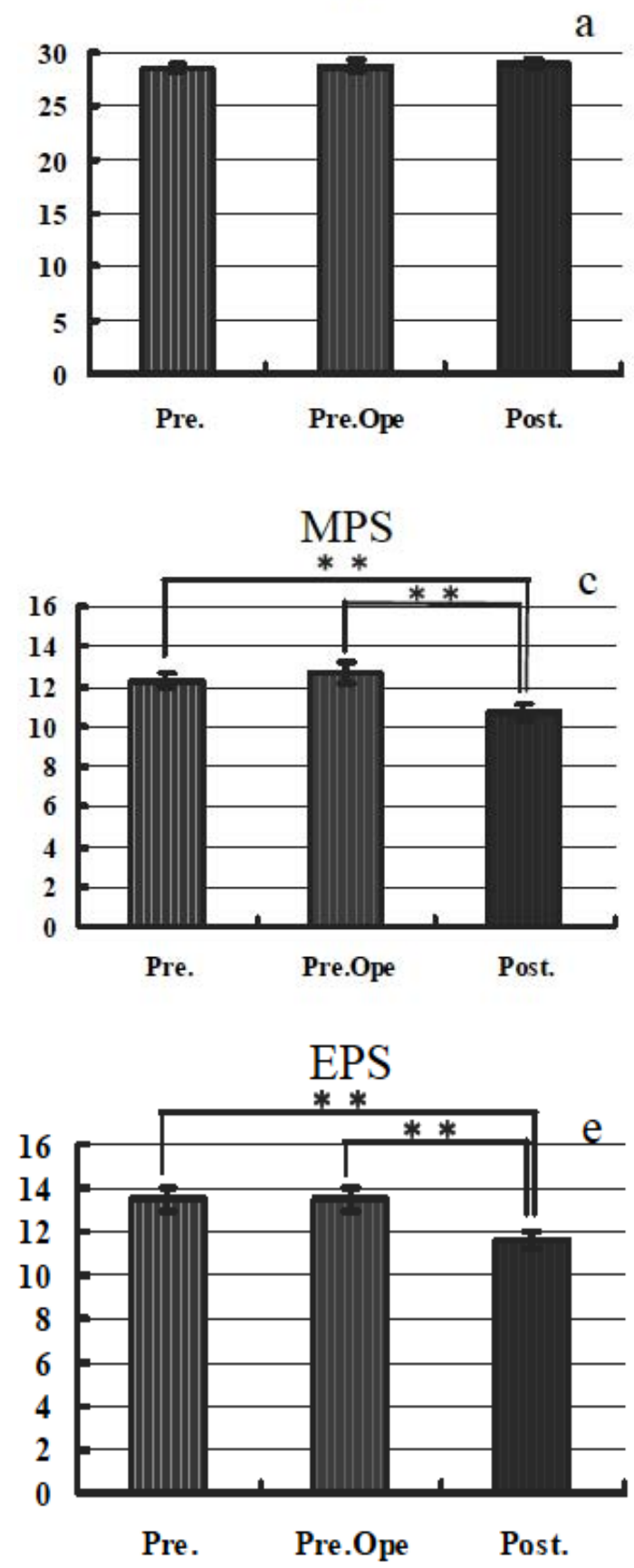

(Figure 3, Table 2).

Difference in the size of the pharyngeal airway before and after treatment in the control group

The size of the pharyngeal airway $(\mathrm{mm})$ before and after treatment was significantly higher only in PPS. However, after adjustment of PPS by analysis of covariance for age and duration of treatment during the initial visit, no significant difference was observed (Figure 4, Table 3).

Difference in the size of the pharyngeal airway before and after
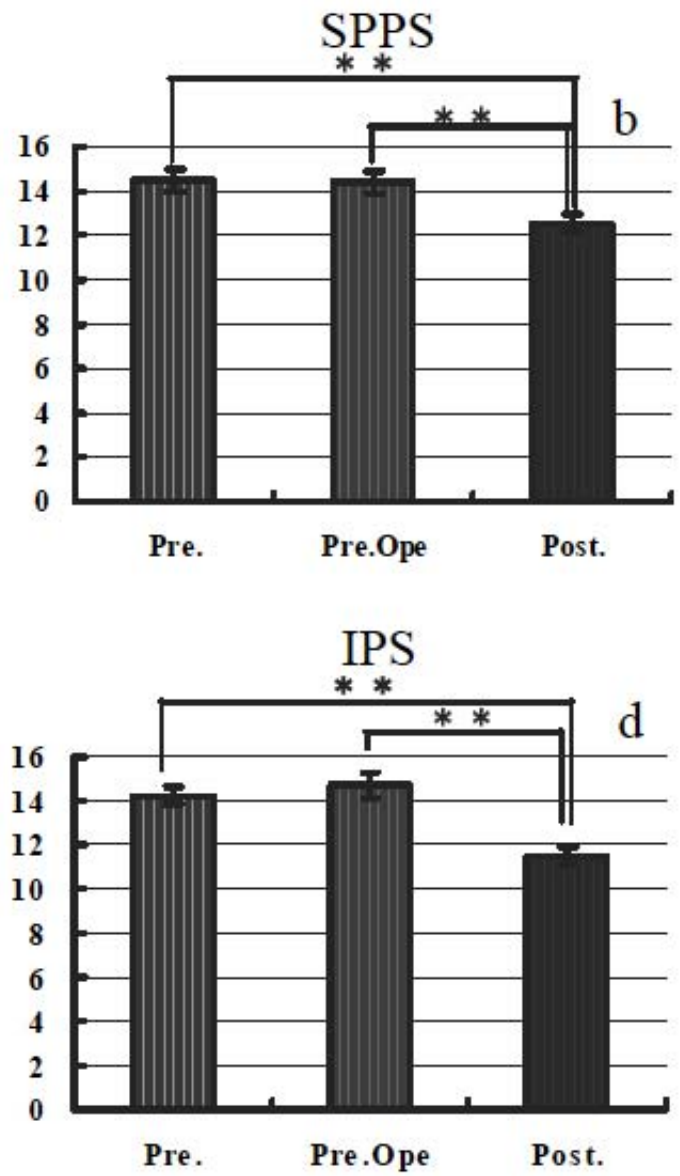

Figure 5. Comparison before and after surgery during the initial visit in the study group One-way repeated ANOVA a-PPS, b-SPPS, c-MPS, d-IPS, e-EPS $\quad * * \mathrm{P}<0.01$

\section{treatment in the study group}

During the initial visit, the size of the pharyngeal airway ( $\mathrm{mm}$ ) before and after surgery, no significant difference in PPS was obtained (Figure 5-a, Table 4). However in SPPS, during the initial visit, there was no significant difference before and after surgery but a significant difference was obtained before and after surgery (Figure 5-b, Table 4). There was a significant difference before and after surgery in MPS (Figure 5-c, Table 4). There was no significant difference before and after surgery in IPS (Figure 5-d, Table 4). A significant difference was observed before and after 
Tomoko Uozumi et al.: Morphological Changes in the Pharyngeal Airway Space

Table 4. Comparison in the study group

\begin{tabular}{llllllll}
\hline & T0: Pre-treatment & T0: Pre-operation & T1: Post-treatment & T0 vs T0' & T0 vs T1 & T0' vs T1 \\
\hline PPS & $28.6 \pm 0.4$ & $28.8 \pm 0.5$ & $28.9 \pm 0.4$ & $\mathrm{p}=0.4736$ & $\mathrm{NS}$ & $\mathrm{NS}$ & $\mathrm{NS}$ \\
SPPS & $14.5 \pm 0.5$ & $14.4 \pm 0.5$ & $12.5 \pm 0.4$ & $\mathrm{p}<0.001$ & $\mathrm{p}=1.00$ & $\mathrm{p}<0.001^{* *}$ & $\mathrm{p}<0.001^{* *}$ \\
MPS & $12.3 \pm 0.4$ & $12.7 \pm 0.5$ & $10.7 \pm 0.4$ & $\mathrm{p}<0.001$ & $\mathrm{p}=0.704$ & $\mathrm{p}<0.001^{* *}$ & $\mathrm{p}<0.001^{* *}$ \\
IPS & $14.2 \pm 0.4$ & $14.7 \pm 0.6$ & $11.5 \pm 0.4$ & $\mathrm{p}<0.001$ & $\mathrm{p}=0.830$ & $\mathrm{p}<0.001^{* *}$ & $\mathrm{p}<0.001^{* *}$ \\
EPS & $13.5 \pm 0.5$ & $13.8 \pm 0.5$ & $11.6 \pm 0.4$ & $\mathrm{p}<0.001$ & $\mathrm{p}=1.00$ & $\mathrm{p}<0.001^{* *}$ & $\mathrm{p}<0.001^{* *}$ \\
\hline
\end{tabular}

surgery in EPS (Figure 5-e, Table 4).

Because of the potential impact in the narrowing of the amount of pharyngeal airway space with a minimum retrusion in SSRO, the groups were divided into 2 with the addition of the median average after surgery in the study group. However, significant differences between the two groups were not observed.

\section{Verification of the hypothesis}

In this study, during the initial visit, a significant difference in the nasopharyngeal level in all parameters was observed between the control group and the study group except for PPS. On the other hand, at the end of active treatment, no significant difference was observed in all parameters. The results suggest that patients with skeletal mandibular protrusion have wider nasopharyngeal airway than those with normal occlusion. This possibility was already mentioned in a report. Tonogi et al. ${ }^{14)}$ studied the changes in sleep-disordered breathing before and after orthognathic surgery. Pharyngeal airway in patients with mandibular prognathism was estimated to be longer and larger compared to healthy patients. Mandibular setback of the jaw can cause low incidence sleepdisordered breathing. Iwasaki et al. ${ }^{15)}$ built a three-dimensional form of child occlusal throat taken at the opposite cone beam CT and reported that the area is larger in comparison with lower pharyngeal airway in children with Class I malocclusion . However, no comparison in post-operative treatment was done. With our clinical experience and based on the results of the study, the possibility of patients with skeletal mandibular protrusion with wider pharyngeal airway than the normal patients was considered.

Based on the results of our study, pharyngeal airway is narrowed from the original wide pharyngeal airway by SSRO which can be comparable to the patient's pharyngeal airway after surgery. It has been previously reported at this point that the reduction of post-operative side effects after orthodontic treatment is significantly different. Nevertheless, in this study, long-term observation after treatment was not done so further studies seems to be necessary.

There was no difference in all parameters between the control group and the study group although narrowing of the pharyngeal airway after surgery was observed in all patients in the study group. Arisaka et al ${ }^{17)}$ concluded that the changes in pharyngeal airway before and after orthognathic surgery is not associated with the changes in sleep-disordered breathing and that other factors such as the change in physiology should be considered. In recent years, obstructive sleep apnea syndrome (OSAS) has been reported to have occurred in patients with mandibular prognathism who underwent mandibular setback surgery. However, recently, it has also been reported to negate the relationship between OSAS and mandibular setback surgery with prognathism ${ }^{14)}$.

Isono $^{18)}$ reported that upper airway muscle activity and airway pressure regardless when stimulated vary dynamically during sleep and the diagnosis and measurement of obstruction in the area is difficult. However, Chen et al. ${ }^{19)}$ considered the various risk factors during surgical correction and predicted the form of pharyngeal airway that is likely to develop OSAS after surgical correction. Comparing this, the development of OSAS has been reported to be an important change in the maxilla ${ }^{17}$. Here we examined a single case of SSRO but further studies of advance cases of orthognathic surgery should be considered.

PPS in case group, during the initial visit, showed no significant change before and after surgery after the active treatment. The same results have been reported post-operatively in patients who underwent mandibular surgery ${ }^{11)}$. However, a significant difference in PPS was observed before and after surgery where narrowing of the oropharyngeal airway below and behind the soft palate occurred. Regarding PPS, in patients with skeletal mandibular protrusion, pharyngeal airway is larger than normal patients but the hypothesis was considered to be not related. However in this study, in the control group, for no apparent reason, there was no significant difference in PPS after treatment.

\section{Subject selection and limitations of the study}

In the present study, since the patients were selected from a large source, a good number of cases were gathered. But because of the special nature of orthodontic treatment and because we could not gather enough male subjects, only female subjects were used in the study. If male patients would have been considered, then the results might be different. 
J.Hard Tissue Biology Vol. 21(1):35-42, 2012

Comparing the number of cases in the study group (53 cases) with the number of control group (28 cases) which is almost less than half, the number of cases was not balanced. This is because selection of cases for the control group in a clinical routine is difficult. However, it was considered later that it was important to increase the number of cases in the control group to increase the reliability of statistical tests and significance.

The patients in the control group were selected from those who had orthodontic treatment alone without skeletal disharmony and not a group without having dynamic orthodontic treatment. Results of this study are therefore intended only for those with orthodontic treatment from a source greater than the normal adult population. Further, the study does not require correction of size of the pharyngeal airway in patients with skeletal mandibular prognathism which cannot be concluded. Because of this, what seems to be needed in the future studies is to have case control study population consisting of non-orthodontic patients matched for age, physique and skeletal mandibular prognathism.

\section{Evaluation of pharyngeal airway}

A two-dimensional cephalogram was used in this study. Cephalometrics was previously used in several studies to measure the pharyngeal airway ${ }^{1,8,11)}$ but it has been recently reported that CT can rapidly provided a three-dimensional data analysis. Kiryu et $\mathrm{al}^{20)}$ reported that building a three-dimensional CT image is qualitatively good even if clinical application is insufficient to quantify for the diagnosis of jaw deformity which cannot be obtained from lateral cehalogram ${ }^{20)}$. However, in recent years, the precise three-dimensional data has been reported to be 4 to 5 times more accurate (accurate within $0.01 \mathrm{~mm}$ ) than twodimensional data ${ }^{21)}$.

If three-dimensional evaluation of this research data were gathered not just antero-posteriorly, more interesting insights would have been obtained. Sugioka et al mentioned that to determine the changes in acute respiratory tract of patients with early post-operative skeletal mandibular prognathism, lateral cepahlometric and three-dimensional CT scan can be performed pre and post-operatively 1 ). Hyoid bone after surgery compensates for the narrowing of the upper airway by moving downward and backward 2), and reported a 22\% decreased airway in the area ${ }^{222}$. Kawamata et al ${ }^{23)}$ reported that three months after surgery, the mean reduction rates of the lateral and frontal widths of pharyngeal airway were $23.6 \%$ and $11.4 \%$, respectively.

In this report, narrowing of the airway 6 months to 1 year after surgery in many cases has occurred and has not recovered. The narrowing of the pharyngeal airway remained consistent with our report. However, because there is no report regarding the comparison of patients with non-surgical orthodontic treatment with healthy subjects, perspectives and approaches to determine the normal state has not yet been elucidated. Considering the report of Kawamata et al. ${ }^{23)}$, the decrease in the two-dimensional airway diameter (antero-posterior diameter) in our study is just a compensation phenomenon of cross-sectional area to ensure the increased in the width but the estimation of the diameter did not occur.

Currently, CT equipment in orthodontic clinics is limited and two-dimensional data is being used on a daily basis. In addition, CT scan has a very high radiation dose compared to lateral cephalogram ,. Considering radiation protection (justification), CT scan in routine clinical practice exceeds the disadvantages such as exposure to carcinogens in which patients will not benefit from the routine use of CT scan for diagnosis.

In conclusion, the ultimate goal of this study was to validate the hypothesis whether pharyngeal airway narrowing occurred in patients after mandibular setback surgery and whether this condition is close to the normal airway. In this study, lateral cephalograms of 53 female adults treated with SSRO (study group) and 28 female adults with completed dynamic orthodontic treatment alone (control group) were examined. Also, for this hypothesis (1) the size of the pharyngeal airway of patients with skeletal mandibular prognathism before surgery and (2) postoperative changes in the pharyngeal airway after surgery were compared. For the results, except for the nasopharynx, the pharyngeal airway in the study group was significantly wider before surgery compared to the control group. However, after treatment, no significant difference was obtained between the two groups. The pharyngeal narrowing in the study group compared to the control group was due to SSRO. The results suggest that patients with skeletal mandibular prognathism have wider pharyngeal airway before surgery which is equivalent to the normal pharyngeal airway.

\section{Acknowledgements}

The authors would like to acknowledge Dr. Takashi Mima, Chief, Department of Dentistry and Oral Surgery, NTT West Osaka Hospital, who performed all the surgical procedured used in this study.

\section{References}

1. Emata K, Mitani H and Sakamoto T. Effect of orthognathic surgery on skeletal mandibular prognathism. Orthod WavesJpn 42: 69-84, 1983

2. Enacar A, Aksoy AU, Sençift Y, Haydar B and Aras K. Changes in hypopharyngeal airway space and in tongue and hyoid bone positions following the surgical correction of mandibular prognathism. Int J Adult Orthodon Orthognath Surg 9: 285-290, 1994

3. Hochban W, Schürmann R, Brandenburg U and Conradt R. Mandibular setback for surgical correction of mandibular hyperplasia-dose it provoke sleep-related breathing 
Tomoko Uozumi et al.: Morphological Changes in the Pharyngeal Airway Space

disorders? Int J Oral Maxillofac Surg 25: 333-338, 1996

4. Achilleos S, Krogstad O and Lyberg T. Surgical mandibular setback and changes in uvuloglossopharyngeal morphology and head posture:A short- and long-term cephalometric study in males. Eur J Orthod 22: 383-394, 2000

5. Gu G,Nagata J, Suto M, Anraku Y, Nakamura K, Kuroe K and Ito G. Hyoid position, pharyngeal airway and head posture in relation to relapse after the mandibular setback in skeletal Class III. Clin Orthod Res 3: 67-77, 2000

6. Turnbull $\mathrm{N}$ and Battagel J. The effects of orthognathic surgery on pharyngeal airway dimensions and quality of sleep. J Orthod 27: 235-247, 2000

7. Liukkonen M, Vähätalo K, Peltomäki T, Tiekso J and Happonen RP. Effect of mandibular setback surgery on the posterior airway size. Int J Adult Orthodon Orthognath Surg 17: 41-46, 2002

8. Yoshida K, Hisanaga Y, Tamaoki S, Kiyosue S and Ishikawa H. Morphological changes in pharyngeal airway and hyoid bone position due to orthognathic surgery of mandibular prognathism. J Fukuoka Dent Coll 30:131-140, 2004

9. Saitoh K. Long-term changes in pharyngeal airway morphology after mandibular setback surgery. Am J Orthod Dentofacial Orthop 125: 556-61, 2004

10. Eggensperger N, Smolka W and Iizuka T. Long-term changes of hyoid bone position and pharyngeal airway size following mandibular setback by sagittal split ramus osteotomy.J Craniomaxillofac Surg 33: 111-117, 2005

11. Kouno K, Igawa K, Takamori K, Kurokawa H and Sakoda S. Morphological changes in pharyngeal airway space after mandibular setback surgery with and without le fort I osteotomy in skeletal class III patients. Jpn J Jaw Deform 16: 184-189, 2006

12. Kitagawara K, Kobayashi T, Goto H, Yokobayashi T, Kitamura N and Saito C. Effects of mandibular setback surgery on oropharyngeal airway and arterial oxygen saturation. Int J Oral Maxillofac Surg 37: 328-333, 2008

13. Foltán R, Hoffmannová J, Pavlíková G, Hanzelka T, Klíma K, Horká E, Adámek S and Sedý J. The influence of orthognathic surgery on ventilation during sleep. Int J Oral Maxillofac Surg 40: 146-149, 2011

14. Tonogi M, Arisaka T, Tukamoto Y, Sato K, Yamane G, Ohkushi T and Nakajima T. Examination of change in sleep-disordered breathing before and after orthognatic surgery. Jpn J Jaw Deform 17: 9-15, 2007

15. Iwasaki T, Hayasaki H,Takemoto Y, Kanomi R and Yamasaki Y. Oropharyngeal airway in children with Class III malocclusion evaluated by cone-beam computed tomography. Am J Orthod Dentofacial Orthop 136: 318-319, e1-318.e9. 2009

16. Mochida M, Ono T, Saito K, Tsuiki S and Ohyama K. Effects of maxillary distraction osteogenesis on the upper-airway size and nasal resistance in subjects with cleft lip and palate. Orthod Craniofacial Res 7: 189-197, 2004

17. Arisaka T, Tonogi M, Tukamoto Y, Sato K, Yamane G, Ohkushi T and Nakajima T. Examination of change in sleepdisordered breathing before and after orthognatic surgeryPart 2 Chang of the pharyngeal airway space. Jpn J Jaw Deform 17: 16-21, 2007

18. Isono S. Diagnosis of sites of upper airway obstruction in patients with obstructive sleep apnea. Nihon Rinsho 58: 96100,2000

19. Chen F, Terada K, Hanada K and Saito I. Predicting the pharyngeal airway space after mandibular setback surgery. J Oral Maxillofac Surg 63: 1509-1514, 2005

20. Kiryu M and Fujita S. Clinical use of three-dimensional images, reconstructed from CT scans of the maxillo-facial region. Jpn J Plast Surg 32: 379-385, 1989

21. Shibazaki R, Chen H, Kubota M, Nakano H and Maki K. Three-dimensional maxillofacial characteristics in skeletal class III asymmetrical jaw deformity cases using cone beam CT. J Showa Univ Dent Soc 26: 39-50, 2006

22. Sugioka S, Kotani J , Momota Y, Sakuma Y, Umemura A, Ueda Y, Kubo Y, Shirasu R, Takahashi I and Kinoshita Z. Acute morphological changes in the upper airway after osteotomy for skeletal mandibular prognathism. J Jpn Dent Soc Anesthesiol 24: 55-61, 1996

23. Kawamata A, Fujishita M, Ariji Y and Ariji E. Threedimensional computed tomographic evaluation of morphologic airway changes after mandibular setback osteotomy for prognathism. Oral Surg Oral Med Oral Pathol 89: 278-287, 2000 
J.Hard Tissue Biology Vol. 21(1):35-42, 2012 